\title{
TU/e emonownen

\section{Nonequilibrium photoluminescence excitation spectroscopy in GaAs: bottleneck and memory effects}

\section{Citation for published version (APA):}

Hannewald, K., Glutsch, S., \& Bechstedt, F. (2003). Nonequilibrium photoluminescence excitation spectroscopy in GaAs: bottleneck and memory effects. Physical Review B, 67(23), 233202-1/4. [233202].

https://doi.org/10.1103/PhysRevB.67.233202

DOI:

10.1103/PhysRevB.67.233202

Document status and date:

Published: 01/01/2003

\section{Document Version:}

Publisher's PDF, also known as Version of Record (includes final page, issue and volume numbers)

\section{Please check the document version of this publication:}

- A submitted manuscript is the version of the article upon submission and before peer-review. There can be important differences between the submitted version and the official published version of record. People interested in the research are advised to contact the author for the final version of the publication, or visit the $\mathrm{DOI}$ to the publisher's website.

- The final author version and the galley proof are versions of the publication after peer review.

- The final published version features the final layout of the paper including the volume, issue and page numbers.

Link to publication

\section{General rights}

Copyright and moral rights for the publications made accessible in the public portal are retained by the authors and/or other copyright owners and it is a condition of accessing publications that users recognise and abide by the legal requirements associated with these rights.

- Users may download and print one copy of any publication from the public portal for the purpose of private study or research.

- You may not further distribute the material or use it for any profit-making activity or commercial gain

- You may freely distribute the URL identifying the publication in the public portal.

If the publication is distributed under the terms of Article 25fa of the Dutch Copyright Act, indicated by the "Taverne" license above, please follow below link for the End User Agreement:

www.tue.nl/taverne

Take down policy

If you believe that this document breaches copyright please contact us at:

openaccess@tue.nl

providing details and we will investigate your claim. 


\title{
Nonequilibrium photoluminescence excitation spectroscopy in GaAs: Bottleneck and memory effects
}

\author{
K. Hannewald, * S. Glutsch, and F. Bechstedt \\ Friedrich-Schiller-Universität Jena, Institut für Festkörpertheorie und Theoretische Optik, Max-Wien-Platz 1, D-07743 Jena, Germany
}

(Received 21 January 2003; published 10 June 2003)

\begin{abstract}
We present a description of photoluminescence excitation (PLE) spectroscopy in GaAs which is not restricted to thermalized carrier distributions but also allows one to treat nonequilibrium electron-hole pairs created by ultrafast femtosecond laser pulses. For small time intervals between pulse excitation and luminescence detection, we predict by means of quantum-kinetic simulations how incomplete relaxation and bottleneck effects lead to significant differences between PLE and absorption spectra which should be easily observable in experiment. The phenomenological assumption of equivalence between PLE and absorption spectra is confirmed only in the long-time limit, i.e., approaching thermal equilibrium.
\end{abstract}

DOI: 10.1103/PhysRevB.67.233202 PACS number(s): 78.55.-m, 63.20.Kr, 71.35.-y, 78.20.Bh

Besides absorption and photoluminescence (PL) experiments, photoluminescence excitation (PLE) measurements are a widely used spectroscopic tool for the characterization of optical transitions in semiconductors. In contrast to the successful development of microscopic theories for absorption (Refs. 1-3) and PL (Refs. 4 and 5) in recent years, an analogous first-principles description of PLE experiments is still missing. Here, we present the first microscopic description of PLE spectroscopy in direct-gap semiconductors such as GaAs.

A typical photoluminescence experiment in semiconductors can be divided into three stages. First, the sample is optically excited out of the ground state by means of a femtosecond laser pulse with a mean photon energy of $\hbar \omega_{p}$. The laser pulse creates electron-hole pairs due to a transfer of electrons from the valence into the conduction band. Second, the nonequilibrium electron and hole distributions relax within their respective bands via energy transfer to the crystal lattice, i.e., a step-by-step excitation of LO phonons in polar semiconductors such as GaAs. And finally, the electron-hole pairs recombine radiatively, resulting in the luminescence process. In PLE spectroscopy, the spontaneous emission from the sample is detected at a fixed photon energy, typically at the lowest excitonic resonance $E_{1 \mathrm{~s}}$ in highpurity samples. The intensity of this signal is then recorded as a function of the pump frequency $\hbar \omega_{p}$. It is usually assumed that the PLE spectrum obtained in this way is roughly equivalent to the ground-state absorption spectrum. A qualitative phenomenological discussion of the relation between PLE and absorption spectra can be found in several textbooks. ${ }^{6,7}$

The supposed equivalence between PLE and absorption signals in semiconductors strongly relies on the assumption that the recombination times are much larger than the intraband relaxation times, i.e., if the laser-excited electrons and holes have enough time to relax completely into quasiequilibrium before radiative recombination. In this case, the emission intensity at the lowest exciton becomes independent of the relaxation rate, and the PLE spectrum can be argued phenomenologically to mimic the absorption signal. ${ }^{6}$ However, this assumption has never been justified by microscopic quantum-kinetic studies. Furthermore, another in- triguing question has remained completely unaddressed so far in the literature: What happens if the time $\Delta t$ between pulse excitation and PL detection is kept shorter than the intraband thermalization times? Then, the one-to-one correspondence between PLE and absorption signals should break down completely, and no simple arguments can be given what the PLE signal looks like. In order to answer these questions, we present here the first nonequilibrium description of PLE spectroscopy.

The PLE theory is developed by means of the following three-step strategy which incorporates the ultrafast nonequilibrium aspects (pulse excitation and intraband relaxation) as well as Coulomb interaction (excitonic effects). First, we determine the time evolution of the electron and hole distributions during pulse excitation and relaxation. This is done by means of electron-LO-phonon quantum-kinetic equations on the level of the second Born approximation ${ }^{8}$ which have previously been employed to describe successfully ultrafast absorption $^{1-3}$ and four-wave-mixing signals ${ }^{9}$ from coherently excited semiconductors. Second, the charge-carrier distributions are converted into spontaneous emission spectra by application of our recently developed Green's function approach to PL in semiconductors. ${ }^{4}$ The key result of this theory can be expressed as an Elliott-like formula for luminescence that allows us to treat arbitrary nonthermal situations. Compared to the alternative method of the so-called "semiconductor luminescence equations" 10 which is based on photon-assisted density matrices, our approach overcomes several shortcomings such as negative PL and overestimated excitonic signals, as demonstrated in Ref. 4. These improvements are especially important because from the calculated PL spectra we need to extract the luminescence intensity at the exciton energy $E_{1 \mathrm{~s}}$. Finally, in the third step, the PLE signals are obtained if the above procedure is repeated varying the pump frequency $\omega_{p}$ while keeping the other parameters (pump intensity, pump pulse length, and lattice temperature) fixed.

For the explicit calculations, we use the bulk GaAs parameters $E_{g}=1.52 \mathrm{eV}$ and $\hbar \omega_{\mathrm{LO}}=36 \mathrm{meV}$. The exciton binding energy is $1 \mathrm{Ry}=E_{g}-E_{1 \mathrm{~s}}=4.7 \mathrm{meV}$, and the homogeneous line broadening is $0.94 \mathrm{meV}$. The lattice temperature 


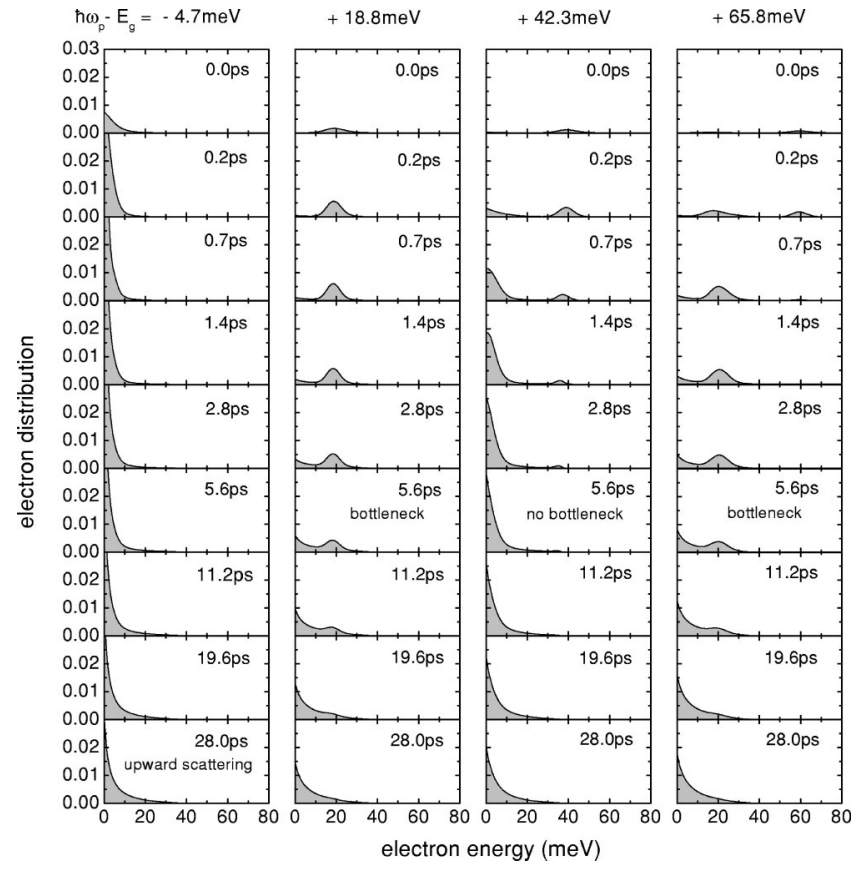

FIG. 1. Electron distribution for times of $0.0,0.2, \ldots, 28.0 \mathrm{ps}$ (from top to bottom) and different detunings of $\hbar \omega_{p}-E_{g}=-4.7$, $18.8,42.3$, and $65.8 \mathrm{meV}$ (from left to right). The pump intensity is $I_{p} \approx 20 \mathrm{~kW} / \mathrm{cm}^{2}$ and the pulse length is $320 \mathrm{fs}$. The lattice temperature is $T=0 \mathrm{~K}$.

is fixed at $T=0 \mathrm{~K}$. The pump pulse is assumed to be Gaussian shaped with a relatively low peak intensity of $20 \mathrm{~kW} / \mathrm{cm}^{2}$ which reduces effects due to screening and electron-electron scattering. The spectral resolution of the luminescence signals is optimized by choosing the pump pulse length to be 320 fs which is significantly larger than the LO-phonon period of $115 \mathrm{fs}$ in GaAs.

In Fig. 1, we present the time evolution of the electron distribution in the conduction band for times of 0.0 , $0.2, \ldots, 28.0 \mathrm{ps}$ (from top to bottom) and different detunings of $\hbar \omega_{p}-E_{g}=-4.7,18.8,42.3$, and $65.8 \mathrm{meV}(-1,4,9$, and $14 \mathrm{Ry}$, from left to right). For excitation high above the band gap, e.g., for $\hbar \omega_{p}-E_{g}=65.8 \mathrm{meV}$, the pump-induced electrons relax within the conduction band through the buildup of phonon replicas of the initial distribution. This initial relaxation process towards the band minimum is relatively fast and takes approximately 1-2 ps. However, the relaxation is not yet complete and the electron distribution is still highly nonthermal. Once the electrons have reached energies below the threshold for LO-phonon emission, the relaxation process drastically slows down and runs into a bottleneck. In fact, in the semiclassical Boltzmann limit with strict energy conservation, such electrons could no longer emit another LO phonon and the distribution would remain nonthermal. However, in quantum mechanics the timeenergy uncertainty principle allows transitions to all energies during the early stages of each scattering event which, in turn, makes further relaxation possible albeit on much longer time scales. Here, after about 25 ps, the electrons are nearly thermalized within the conduction band whereas the holes (not shown) still exhibit a minor bottleneck effect.

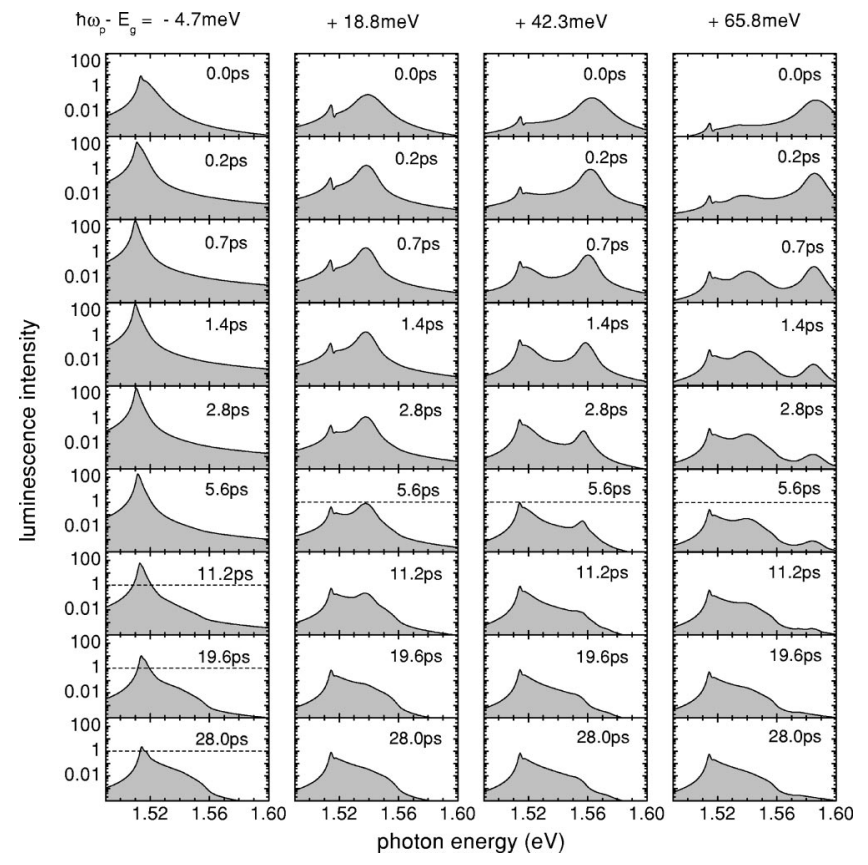

FIG. 2. Luminescence spectra for the same times and parameters as in Fig. 1. The dashed lines are guides for the eye. For an explanation, see text.

The strength of the bottleneck effect depends on the pump frequency of the exciting laser pulse. More strictly, the intraband relaxation may experience only a weak bottleneck effect if the detuning $\hbar \omega_{p}-E_{g}$ is approximately equal to an integer multiple of $\left(1+m_{e} / m_{h}\right) \hbar \omega_{\mathrm{LO}}=41.5 \mathrm{meV}$ where the prefactor $\left(1+m_{e} / m_{h}\right)$ accounts for the fact that the excess energy is split up between the electrons and holes according to their mass ratio. The correlation between the strength of the bottleneck and the detuning is exemplified in Fig. 1 for $\Delta t=5.6 \mathrm{ps}$. While for $\hbar \omega_{p}-E_{g}=42.3 \mathrm{meV}$, i.e., when the above "resonance" condition is fulfilled, all electrons have already assembled near the conduction-band minimum after $5.6 \mathrm{ps}$, the distributions for $\hbar \omega_{p}-E_{g}=18.8 \mathrm{meV}$ and 65.8 $\mathrm{meV}$ are still highly nonthermal.

In Fig. 2, we show the logarithmic-scale plots of the PL spectra corresponding to the distributions of Fig. 1. The effects described above are nicely seen in the emission signals. For excitation high above the band gap, e.g., for $\hbar \omega_{p}-E_{g}$ $=65.8 \mathrm{meV}$, we observe a transfer of the PL intensity from the initial signal at the pump frequency towards lower energies via phonon replicas. Furthermore, the relaxation process is accompanied by a gradual buildup of PL at the excitonic resonance, which becomes the dominant contribution after about 2 ps, in accordance with the fast initial scattering towards the band minimum. The bottleneck effects are still visible in the PL signal at $5.6 \mathrm{ps}$ as pronounced peaks at approximately $1.54 \mathrm{eV}$ and $1.59 \mathrm{eV}$ due to the incomplete electron and hole relaxation, respectively. On longer time scales, when the bottleneck is slowly removed, these structures are smeared out while at the same time the excitonic emission, relevant for PLE, is continuously increasing.

The dependence of the bottleneck strength on the pump detuning is also reflected in the PL spectra, as visualized in 


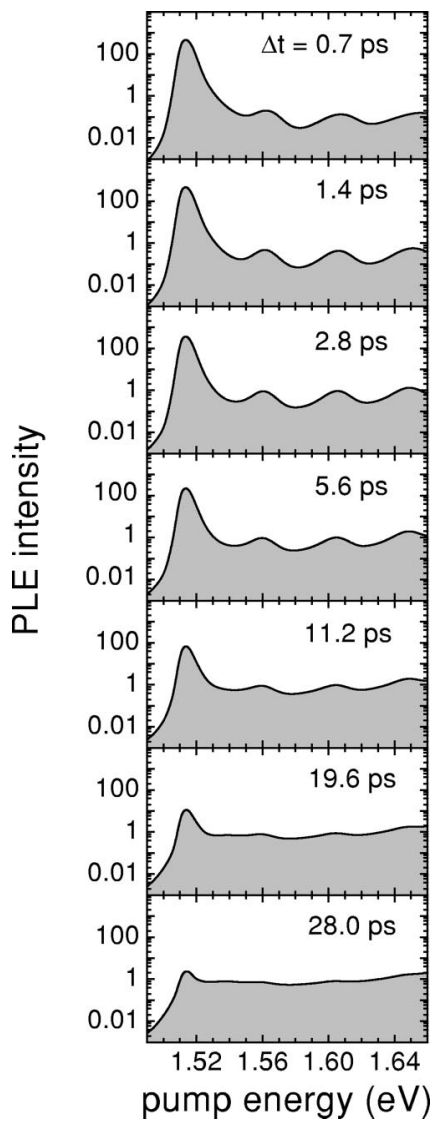

FIG. 3. PLE intensity vs pump energy $\hbar \omega_{p}$ for time differences $\Delta t=0.7,1.4, \ldots, 28.0 \mathrm{ps}$ between pulse excitation and PL detection. Other parameters as in Figs. 1 and 2.

Fig. 2, again for $\Delta t=5.6 \mathrm{ps}$. From a comparison of the three spectra for positive detunings, it can be seen that the PL signal at the excitonic resonance is largest for $\hbar \omega_{p}-E_{g}$ $=42.3 \mathrm{meV}$, i.e., when the bottleneck is very weak. Even for a lower pump frequency such as, e.g., $\hbar \omega_{p}-E_{g}$ $=18.8 \mathrm{meV}$, the excitonic emission, relevant for PLE, is weaker due to the stronger bottleneck in the carrier relaxation.

Now, we proceed with the calculation of the time evolution of the PLE spectra resulting from the variation of the pump frequency $\omega_{p}$. More precisely, the PLE spectra are obtained by extracting the luminescence intensities at the excitonic peaks from Fig. 2 (and from analogous calculations for the other pump frequencies). The results are plotted in Fig. 3. It follows that the PLE spectrum significantly depends on the time interval $\Delta t$ between pulse excitation and PL detection. For short intervals up to a few picoseconds, the PLE spectra are not similar at all to the linear absorption but strongly dominated by the signal at the exciton which is about three orders of magnitude larger than the PLE intensity above the band gap. This is due to the fact that for high pump frequencies the excited electrons and holes have not yet completely relaxed towards their band minima, as demonstrated in Fig. 1. As a consequence, the emission is not solely from the exciton (cf. Fig. 2), but by definition only the excitonic emission contributes to the PLE signal. Furthermore, there
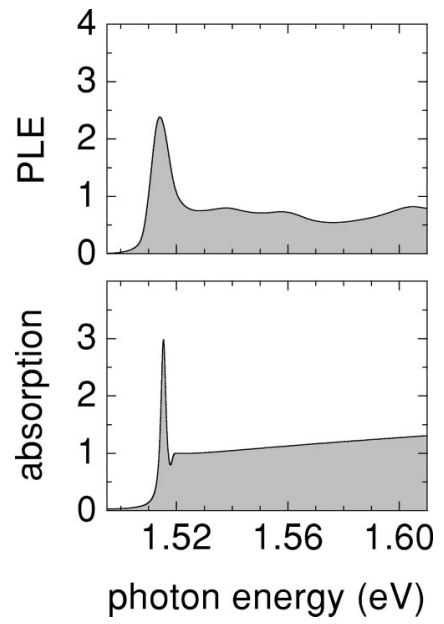

FIG. 4. PLE spectrum for $\Delta t=28$ ps (top) vs ground-state absorption spectrum (bottom). The $y$ axes are individually normalized.

are pronounced satellite peaks in the continuum region which are completely absent in the linear absorption spectrum. They clearly reflect the bottleneck effects described above. Depending on the pump detuning $\hbar \omega_{p}-E_{g}$, the intraband relaxation may experience either a weak or a strong bottleneck effect, which in turn gives rise to peaks and dips, respectively, in the PLE signals at early times. It is worthwhile to mention that for the more polar II-VI semiconductors such as $\mathrm{ZnSe}$ similar LO-phonon cascades have been observed in stationary PLE experiments and were interpreted as hot-carrier effects. ${ }^{11,12}$

In the long-time limit, the picture changes dramatically and the PLE spectrum resembles much more the absorption spectrum. Since the bottleneck effects are slowly removed, the dips in the satellite structures become less and less pronounced and at $\Delta t=28.0 \mathrm{ps}$ the phonon replicas are almost totally smeared out. Furthermore, the ratio between the PLE signal at the exciton and the continuum has become almost the same as in the absorption signal because the PLE signal from the continuum is one order of magnitude larger than for $\Delta t=0.7 \mathrm{ps}$ whereas the PLE signal from the exciton has decreased strongly by two orders of magnitude. While the first effect can again be attributed to the removal of the bottleneck effect on long time scales, the behavior for $\hbar \omega_{p} \approx E_{1 \mathrm{~s}}$ is quite unexpected but can be explained by an analysis of the time evolution of the charge-carrier distributions under these pump conditions. This is done in the left column of Fig. 1 which shows the electron distributions for $\hbar \omega_{p}-E_{g}$ $=-4.7 \mathrm{meV}(-1 \mathrm{Ry})$, i.e., for $\hbar \omega_{p}=E_{1 \mathrm{~s}}$. Again, scattering processes without energy conservation turn out to play an important role. Here, after the excitation, the pump-induced electrons and holes occupy initially only a very narrow region close to the band extrema. In the semiclassical limit, these charge carriers would not experience any intraband relaxation at all at zero temperature. However, this is no longer true in quantum mechanics where the time-energy uncertainty principle renders a possibility for upward scattering even in the limit $T=0 \mathrm{~K}$. As a consequence, the electrons and holes are gradually redistributed to higher kinetic energies. In turn, this leads to a strong reduction of the PL signal 
at the excitonic resonance, as shown in the left column of Fig. 2, and explains the significant decrease in the excitonic PLE signal seen at large $\Delta t$ in Fig. 3.

We conclude our studies by a direct comparison between the absorption spectrum and the PLE signal in the long-time limit $(\Delta t=28 \mathrm{ps})$. From Fig. 4, the resemblance between both spectra is obvious and provides a microscopic justification of the phenomenological assumption of equivalence between PLE and absorption. Nevertheless, we observe some slight deviations: the PLE spectrum exhibits a broader exciton line and small but noticeable modulations in the continuum signal. As explained above, the latter effect is related to bottleneck effects and we expect it to vanish on nanosecond time scales, especially when the scattering with acoustic phonons (not considered here) becomes important, too. The other effect, the line broadening of the exciton in PLE, is mainly determined by the spectral width of the pump pulse, in contrast to the excitonic linewidth in absorption which is only subject to dephasing, i.e., homogeneous line broadening. However, we find also that the exciton in the PLE signal is much better resolved than one would expect from a con- volution of the absorption spectrum with the pump-pulse spectrum where the exciton is strongly broadened (not shown).

In conclusion, we have presented a novel description of photoluminescence excitation spectroscopy in GaAs. The theory is not restricted to thermal equilibrium but also allows the treatment of highly nonequilibrium systems such as optically excited electron-hole pairs created by ultrafast femtosecond laser pulses. The phenomenological assumption of equivalence between PLE and absorption spectra is confirmed in the long-time limit, i.e., approaching thermal equilibrium. For small time intervals between excitation and luminescence detection, we predict from quantum-kinetic simulations how incomplete relaxation and bottleneck effects influence the PLE signals. The resulting characteristic signatures in nonequilibrium PLE spectra should be easily observable in experiment since the setup can be customized to require only picosecond time resolution. Thus, our findings offer a new possibility for time-resolved studies of hotcarrier phenomena by means of emission spectroscopy.
*Present address: Department of Applied Physics, Eindhoven University of Technology, P.O. Box 513, 5600 MB Eindhoven, The Netherlands.

${ }^{1}$ C. Fürst, A. Leitenstorfer, A. Laubereau, and R. Zimmermann, Phys. Rev. Lett. 78, 3733 (1997).

${ }^{2}$ A. Schmenkel, L. Bányai, and H. Haug, J. Lumin. 76\&77, 134 (1998).

${ }^{3}$ K. Hannewald, S. Glutsch, and F. Bechstedt, Phys. Rev. B 61, 10792 (2000).

${ }^{4}$ K. Hannewald, S. Glutsch, and F. Bechstedt, Phys. Rev. B 62, 4519 (2000).

${ }^{5}$ K. Hannewald, S. Glutsch, and F. Bechstedt, Phys. Rev. Lett. 86, 2451 (2001).

${ }^{6}$ P.Y. Yu and M. Cardona, Fundamentals of Semiconductors
(Springer, Berlin, 1999).

${ }^{7}$ C.F. Klingshirn, Semiconductor Optics (Springer, Berlin, 1997).

${ }^{8}$ J. Schilp, T. Kuhn, and G. Mahler, Phys. Rev. B 50, 5435 (1994).

${ }^{9}$ L. Bányai, D.B. Tran Thoai, E. Reitsamer, H. Haug, D. Steinbach, M.U. Wehner, M. Wegener, T. Marschner, and W. Stolz, Phys. Rev. Lett. 75, 2188 (1995).

${ }^{10}$ M. Kira, F. Jahnke, and S.W. Koch, Phys. Rev. Lett. 81, 3263 (1998).

${ }^{11}$ R.P. Stanley and J. Hegarty, in Optics of Semiconductor Nanostructures, edited by F. Henneberger, S. Schmitt-Rink, and E.O. Göbel (Akademie Verlag, Berlin, 1993).

${ }^{12}$ H. Kalt, M. Umlauff, J. Hoffmann, W. Langbein, J.M. Hvam, M. Scholl, J. Söllner, M. Heuken, B. Jobst, and D. Hommel, J. Cryst. Growth 184\&185, 795 (1998). 